\title{
SotonAUV: the design and development of a small, manoeuvrable autonomous underwater vehicle
}

\author{
J Akhtman \\ School of Electronics and Computer Science, University of Southampton, UK \\ M Furlong \\ National Oceanography Centre, Southampton, UK \\ A Palmer, A Phillips, SM Sharkh and SR Turnock \\ School of Engineering Sciences, University of Southampton, UK
}

\begin{abstract}
The University of Southampton's entry into the Student Autonomous Underwater Challenge - Europe (SAUC-E) 2007 was a custom designed and built autonomous underwater vehicle (AUV) named 'SotonAUV'. Originally developed for SAUC-E 2006, the vehicle was significantly upgraded for the 2007 competition. The mechanical design of the vehicle is described, and an overview of the autonomy and control approaches employed is provided. The updated vehicle successfully competed in SAUC-E 2007, winning first place in the overall competition and taking the BAE Systems prize for innovation in autonomy.
\end{abstract}

Keywords: autonomous underwater vehicle (AUV), SotonAUV, Student Autonomous Underwater Challenge - Europe (SAUC-E), low-level control, submarine, graduate education

\section{Introduction}

The Student Autonomous Underwater Challenge - Europe (SAUC-E) is a competition for student teams and aims to "encourage young engineers and scientists to think about underwater technology and its future possibilities, as well as fostering ties between the students and the organisations involved in AUV technologies' (DSTL, 2007). The second annual SAUC-E competition was held in July 2007 at QinetiQ's Ocean Basin at Haslar, Gosport, UK. This attracted six entries; three returning teams from the 2006 competition from the Universities of Bath, Heriot-Watt and Southampton, and three new teams from the Universities of Cambridge and Nice and the Ecole Nationale Supérieure des Ingénieurs des Etudes et Techniques d'Armements (ENSIETA, Brest).

This briefing provides an overview of the design of SotonAUV, including the mechanical design and the control and autonomy approaches implemented on the vehicle. The lessons learnt from the design of SotonAUV were used as the basis of a new vehicle capable of operating in saltwater. The purpose of these low-cost vehicles is to provide design and operational information for the next generation of long-range AUVs under development at the National Oceanography Centre, Southampton.

\section{Vehicle overview}

SotonAUV was originally developed by a group of eight final-year Master of Engineering undergraduate students from the University of Southampton, for the 2006 SAUC-E competition (see Fig 1). The design philosophy was for a low mass $(<25 \mathrm{~kg})$ AUV with good hydrodynamic performance to simplify low-level control. Previous experience has demonstrated a need for a robust design, with an emphasis on design for maintenance.

The basic vehicle consists of a hydrodynamic composite shell with a rounded rectangular crosssection, which is $1.31 \mathrm{~m}$ long, $0.26 \mathrm{~m}$ wide and $0.22 \mathrm{~m}$ deep. The shell is attached to an internal frame constructed using a lightweight aluminium extrusion. The central part of the frame supports a pair of pressure vessels with extensions to provide mountings for the thrusters, cameras (one forward-looking, one downward-looking and one upward-looking), a kill switch and a marker dropping mechanism.

\subsection{Propulsion and control}

Propulsion and control is provided by two wing-mounted side thrusters and two through-body vertical tunnel thrusters. The thrusters are mounted onto the frame using bespoke mountings. The particular thrusters used are rim-driven (Sharkh et al., 2004) and offer symmetrical performance simplifying the controller design. The thruster layout provides control over surge, heave, pitch and yaw motions. The hydrodynamic stability of the 


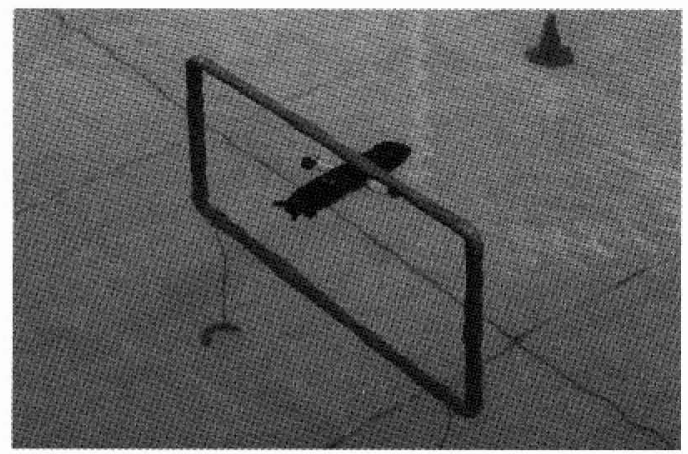

Fig 1: SotonAUV passing through the validation gate at SAUC-E 2007

vehicle is enhanced by a cruciform of fixed control surfaces mounted at the stern.

\subsection{Pressure vessels}

The pair of central pressure vessels is divided into a battery pressure vessel and an electronics pressure vessel. The battery pressure vessel is a commercial acrylic box modified to accommodate connections using cable glands. The $12 \mathrm{~V} 7 \mathrm{Ah}$ battery pack consists of a single sealed lead acid module of six cells providing around $20 \mathrm{~min}$ of operation.

The electronics pressure vessel is an acrylic tube with two aluminium end caps. One end cap is glued in place and the other has an O-ring seal. The latter end cap is equipped with wet-mateable underwater connectors and cable glands to provide connections with the externally mounted components and the battery pressure vessel. The pressure vessel houses the sensor payload including a magnetometer, a pressure sensor and an inertial measurement unit. These sensors are connected to the main computer via a USB data acquisition system. The thrusters are controlled using four elẹctronic speed controllers connected to the main computer via a USB servo controller board. The main computer is a Kontron 986 Mini-ITX motherboard with a $2 \mathrm{GHz}$ Core Duo processor and $2 \mathrm{~GB}$ of RAM. This system was chosen for its small form factor $(170 \times 170 \mathrm{~mm})$, high computing power and device connectivity having eight USB and four RS232 ports.

\section{Autonomy and control}

The control software was developed in Matlab with Windows XP as the operating system. The advantages of this approach are that it is simple to interface with the external components, and the software can be rapidly developed using the in-built toolboxes within Matlab. The disadvantages are, firstly, that Windows XP is not a real-time operating system, hence hard real-time constraints cannot be guaranteed, and secondly, that interpreted Matlab is not computationally efficient. However, the available processing power and the low speed of the vehicle $\left(<0.5 \mathrm{~m} . \mathrm{s}^{-1}\right)$ meant that this does not greatly affect the vehicle performance.

\subsection{Control software}

The control software developed for the AUV consists of a number of hierarchical structured modules. At the highest level, the mission controller is responsible for the overall mission management. It takes processed sensor data and compares it to the mission objectives before outputting demands to the low-level controller. Below the mission controller sits the navigation and image processing modules. These modules are responsible for processing the image data into target data and navigation information before feeding the results to the mission controller. Finally, the low-level control runs modules that command the depth, pitch, heading and forward speed of the vehicle.

The modules forming the control software are an ensemble of software agents (SAs), with each SA constituting an independent instance of Matlab and being responsible for a different aspect of the vehicle's autonomous behaviour. The SAs run in parallel, while constantly exchanging information using User Datagram Protocol (UDP) signalling (Postel, 1980). The main advantages of a control system comprising multiple SAs, as opposed to a single process-based control scheme, may be summarised as follows:

- Parallelism: The computationally intensive processes can run in the background without interfering with the essential real-time control processes with the operating system performing the necessary multitasking.

- Efficiency: The parallel processing makes optimal use of the available computational resources.

- Scalability: SAs can run on any number of independent hardware components.

- Robustness: The overall control system can recover in the case of failure of a certain number of SAs.

The control software consists of a system of five SAs with a structured query language (SQL) database, which can also be considered as an SA. The particular SAs are a mission controller, a motion controller, two camera agents and a remote control and monitoring agent. Splitting the control software into these conceptual modules simplified the development process allowing each element to be developed separately.

\subsection{Mission controller}

The AUV mission is separated into a number of sequential tasks within the mission controller. Each task is attempted by the AUV in turn, and success or 
failure is determined before stepping onto the next task. The tasks are considered successful if the AUV has met a 'Pass' criterion (for example, diving to a set depth), or are considered unsuccessful if a 'Fail' criterion is met or if a time-out is reached. Once the pass, fail or time-out event has occurred, the mission controller steps onto the next task. This approach allows the AUV to attempt all tasks.

To simplify the implementation of the tasks, a number of macros have been developed that can be used repeatedly within a mission. An example of this is searching for a mid-water target. Here, the AUV maintains constant depth and pitch whilst turning in regular angular increments up to $360^{\circ}$ and capturing images from the forward-looking camera. Each image is analysed to determine if the mid-water target is in the frame, and the macro terminates when either the target has been found, or the AUV has turned through $360^{\circ}$. For searching a large area, this operation can be repeated at multiple equi-spaced locations, with the increment between the locations dependent on the clarity of the water. While controlling a particular task, the mission controller determines the necessary change in motion of the AUV and sends depth, heading and speed demands to the low-level controller.

\subsection{Navigation and image processing}

The navigation capability of SotonAUV relies largely on simultaneous localisation and mapping (SLAM), accomplished through real-time processing of the visual images of the tank (sea) floor. More specifically, the vehicle employs the mapping technique outlined in Eustice (2005), where the visual map of the relevant region is incrementally acquired using the sequence of snap-shots taken from the downward-looking camera. Each consecutively acquired image is registered using a fast Fourier transform (FFT) based method (Porat, 1997). Firstly, the newly acquired image is correlated with the preceding image. The partial overlap between the two images allows the estimation of the incremental translation, scaling and rotation of the new image compared to the previous one. Secondly, the estimates of the incremental changes between the two consecutive images are utilised in order to position the newly acquired image in the context of the available map consisting of the data extracted from all previously acquired images. Finally, the extrinsic information from the new image is utilised to extend the mapped area and to calculate any change in the vehicle's global coordinates.

The images from the forward-looking camera are utilised for target reconnaissance, obstacle avoidance and mapping. Each consecutive image is scanned for features having a convex shape and homogeneous colour, where the colour is defined as the 'hue' constituent of the hue-saturation-value (HSV) colour space (Porat, 1997). Subsequently, any feature identified as a potential target is analysed and classified using predefined sets of shape and colour properties associated with the objects relevant to the planned missions. (Note that all the underwater objects were characterised as part of the competition rules.)

For example, a red spherical target is defined as a round blob having an average hue of $H=0$, with a roundness metric defined as $4 \pi A / P^{2}$ (where $A$ is the target area and $P$ is the target perimeter). Once a target is identified with a high level of confidence (the corresponding metric exceeds a given threshold), the mission-planning module calculates an appropriate adjustment to the desired heading and depth values. In the case of target tracking, the adjustments are aimed at bringing the centre of mass of the target to the centre of the field of view of the forward-looking camera. In the case of obstacle avoidance, however, the heading adjustments are aimed at minimising the probability of collision, while maintaining the tracking of any potential targets. Furthermore, for each detected target, an estimate of the target's coordinates are calculated and employed for both mapping and mission planning, particularly tracking of targets outside of the instantaneous field of view.

\subsection{Low-level control}

The low-level control is split into a vertical controller and a horizontal controller, which act independently of each other. The vertical controller uses simple proportional control for depth, with a bias in the thruster demands based on the vehicle pitch. The horizontal controller sends demands to the horizontal thrusters, depending upon the required speed, with a bias in the demand values based on the heading error of the vehicle.

\section{Concluding remarks}

During 2007, a significant upgrade of SotonAUV was undertaken to improve the vehicle for the 2007 SAUC-E competition. A new propulsion system was installed, a new pressure vessel was designed and manufactured, and the control software was significantly updated.

SotonAUV was entered into the 2007 SAUC-E competition held on 12-15 July 2007 at the $5.5 \mathrm{~m}$ deep Haslar Ocean Basin, Gosport, UK. During the in-water trials, the vehicle successfully qualified for the final round by passing through the validation gate and then produced the most successful run in the final round. During this run, SotonAUV successfully navigated its way through the validation gate, dropped a marker on the circular target, 
located - but narrowly missed - the mid-water target and surfaced within the surfacing zone. This successful run, coupled with the static assessment of the vehicle, meant that the University of Southampton team was awarded first place in the overall competition, winning the prize sponsored by QinetiQ. It was also awarded the BAE systems prize for innovation in autonomy. The University of Cambridge team placed second overall and the ENSIETA team took third place. The ENSIETA team were also awarded the Thales prize for innovation in control.

The challenge posed by the SAUC-E competition has stimulated the development of a new low-cost AUV. The vehicle development formed the basis of a high-quality Group Design Project for final-year engineering students in 2006 and, in 2007, was enhanced to provide a platform for research by the team of PhD and EngD students.

\section{Acknowledgements}

The University of Southampton team would like to thank its many sponsors including: National
Oceanography Centre, Southampton; School of Engineering Sciences (SES), University of Southampton; Engineering and Physical Sciences Research Council; TSL Technology Ltd for the loan of four $70 \mathrm{~mm}$, rim driven thrusters; Kontron for donating the Mini-ITX boards; REAP Systems Ltd for providing battery systems and SES Technicians for their skilled support.

\section{References}

Defence Science and Technology Laboratory (DSTL). (2007). DSTL website for SAUC-E, http://www.dstl. gov.uk/news_events/competitions/sauce/index.php, accessed on 4 October 2007.

Eustice R. (2005). Large-Area Visually Augmented Navigation for Autonomous Underwater Vehicles. Massachusetts Institute of Technology/Woods Hole Oceanographic Institution Joint-Program, PhD Thesis.

Porat B. (1997). A Course in Digital Signal Processing. London: Wiley.

Postel J. (1980). User Datagram Protocol, USC/Information Sciences Institute, $R F C \mathbf{7 6 8}$.

Sharkh SM, Lai SH and Turnock SR. (2004). Structurally integrated brushless PM motor for miniature propeller thrusters. IEE Proceedings - Electric Power Applications 151: 513-519. 